\title{
Wie kann Ernährung die Frakturheilung fördern?
}

\author{
Harun Seyhan
}

In der Vergangenheit wurde in vielerlei Facetten über die wichtige Rolle der Ernährung in Bezug auf die Knochenheilung publiziert. Sogar Verhaltensregeln wurden aufgestellt. Doch wie ist der aktuelle Stand?

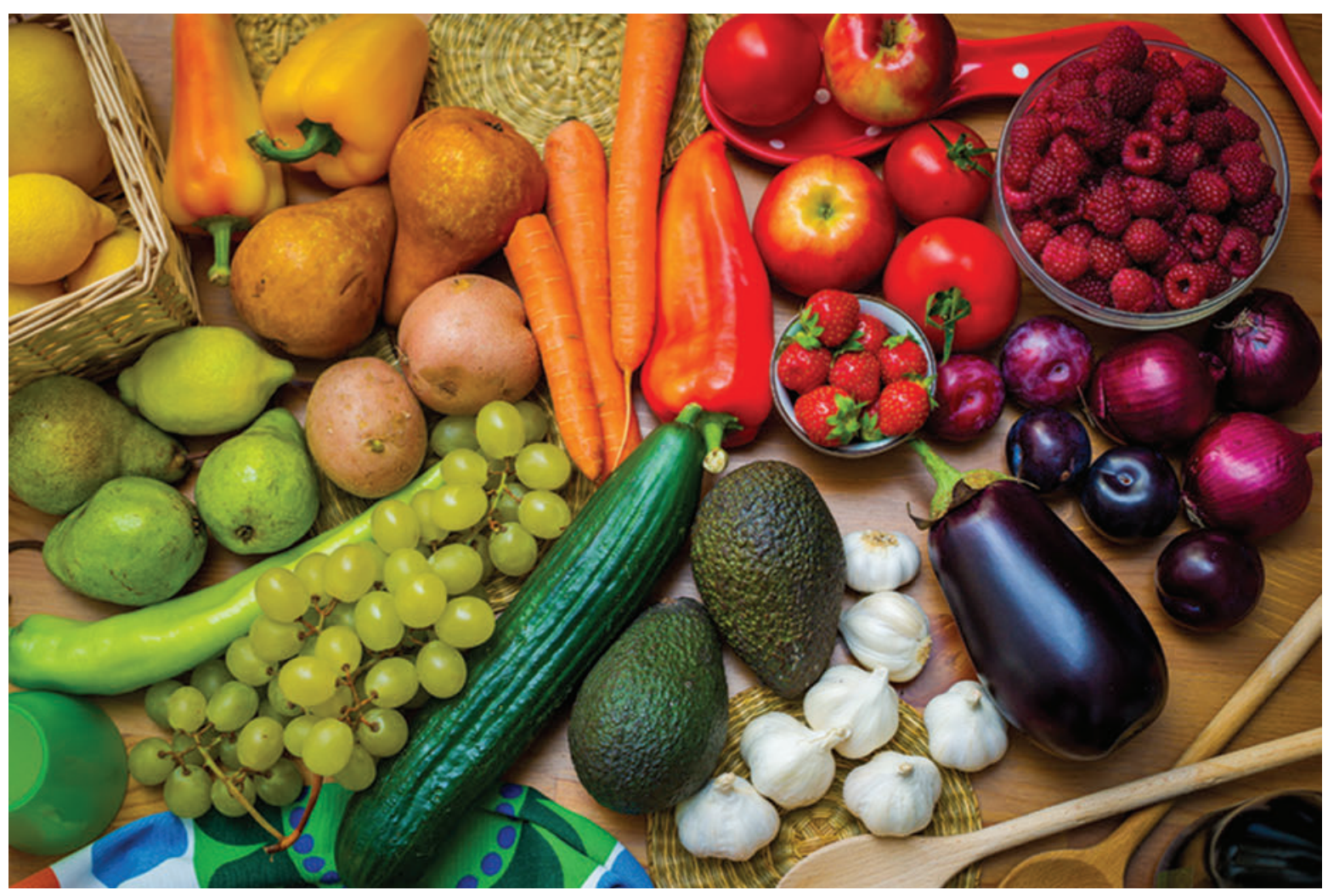

Gesunde Ernährung unterstützt die Bruchheilung. (Quelle: napocska/Shutterstock.com)

\section{Kalzium}

Es bildet den wichtigsten Knochenbaustein. 99\% des Kalziums in unserem Körper sind im Skelett gebunden. Liegt ein Mangel vor, wird Kalzium aus dem Knochen freigesetzt, was allmählich zulasten dessen Festigkeit geht [1]. Häufig fand das Zusammenspiel zwischen Kalzium und später auch Vitamin D in der Literatur Erwähnung. Die konsequente Einnahme/Substitution durch Milch und Milchprodukte sowie Cholecalciferol oder Sonnenbäder wurde empfohlen. Neue Erkenntnisse belegen, dass Kalzium sehr gut durch dunkelgrünes Gemüse, Wildpflanzen, Nüsse (wie beispielsweise Mandeln, Haselnüsse, Walnüsse), Samen, Früchte (wie Schwarze
Johannisbeere, Feigen, Kokosnuss, Apfel, Birne, Mango, Bananen, Avocado) und diverse Mineralwassersorten vom Körper aufgenommen werden kann.

\section{Magnesium}

Der Organismus braucht neben Kalzium die verschiedensten Mineralstoffe und Spurenelemente, wie z. B. Zink, Bor und Kupfer. Ein für die Knochen ganz besonders wichtiger Mineralstoff ist das Magnesium, da das Kalzium nur mit Magnesium wirken kann. Dabei brauchen die Knochen das richtige Kalzium-Magnesium-Verhältnis. So wird empfohlen, Magnesium und Kalzium in einem Verhältnis von 2:1 (Kalzium zu Magnesium) einzunehmen, um von beiden 
Mineralien und ihren positiven Wirkungen optimal zu profitieren. Gute Magnesiumlieferanten sind z. B. Haferflocken, Salat, Spargel, Vollkorngetreide, Kürbis, Kartoffel, Pfirsich, Erbsen, Linsen, Pflaumen und natürlich Nüsse (Haselnüsse, Walnüsse).

\section{GUT ZU WISSEN!}

Verhältnis Kalzium zu Magnesium:

- Kuhmilch 10:1

- Käse bis 30:1

- Sango Meereskoralle $2: 1$

Die empfohlene tägliche Mindestdosis an Magnesium liegt für Frauen bei $320 \mathrm{mg}$ und für Männer bei $400 \mathrm{mg}$.

\section{Vitamin D}

Es ist eines der wenigen Vitamine, die der Mensch selbst bilden kann. In der Regel entwickelt der Körper in der Haut 80-90\% des Vitamins mithilfe von Sonnenlicht, genauer UV-B-Strahlung. Vitamin D steuert die Aufnahme (Resorption) von Kalzium und Phosphat aus dem Darm sowie ihren Einbau in den Knochen. Es nimmt damit eine Schlüsselrolle bei der Knochenmineralisierung ein. Die Ernährung trägt mit einem geschätzten Anteil von circa 10-20\% nur einen relativ geringen Anteil zur Vitamin-D-Versorgung bei. Ein Grund hierfür ist, dass nur wenige Lebensmittel nennenswerte Mengen an Vitamin D enthalten (z. B. fetter Seefisch, bestimmte Innereien, Speisepilze, Eier). Ein Vitamin-D-Mangel kann zur Entkalkung und letztlich zur Erweichung der Knochen führen und damit zum Krankheitsbild der Osteomalazie. Eine weitere Erkrankung, zu der ein Vitamin-D-Mangel beitragen kann, ist Osteoporose, die sich vor allem im höheren Lebensalter manifestiert. Sie ist durch eine erniedrigte Knochenmasse sowie eine mikroarchitektonische Verschlechterung des Knochengewebes gekennzeichnet, was zu einer geringeren Bruchfestigkeit des Knochens führt.

\section{Vitamin $\mathrm{K}$}

Es ist ebenfalls aktiv am Knochenaufbau beteiligt. Erst unter seiner Einwirkung kann im Knochen das Protein Osteocalcin gebildet werden, das Kalzium bindet und für einen gesunden Knochenaufbau zuständig ist. Grüne Gemüse und Kräuter sind besonders ergiebige VitaminK-Quellen, z. B. Brokkoli, Blumenkohl, Rosenkohl, Spinat, Portulak, Schnittlauch, Petersilie, Blattsalate und besonders der Grünkohl.

\section{Vitamin C}

Vitamin C (Ascorbinsäure) ist gut für die Synthese von Kollagen. Es stimuliert die knochenaufbauenden Zellen und verbessert die Aufnahme von Kalzium. Vitamin C kommt in nahezu allen pflanzlichen Lebensmitteln vor. Besonders reich an Vitamin $C$ sind folgende Obst- und Gemüsesorten: Sanddornbeeren, Paprika, Kohl (z. B.
Kohlrabi, Grünkohl, Rosenkohl), Zitrusfrüchte, Beerenfrüchte, Spinat.

Die Knochen bestehen in der Hauptsache aus Kalzium, Phosphor, Magnesium, Kalium und Silizium. Wenn sich diese Mineralstoffe mit Kollagenfasern verbinden, spricht man von der Mineralisierung des Knochens. Der Knochen wird aufgebaut und verhärtet sich, ohne jedoch seine erforderliche Biegsamkeit zu verlieren. Das Silizium dabei ist wichtig für die Kollagenfasersynthese und auch für den Mineralisierungsprozess des Knochens. Hilfreiche Siliziumquellen sind beispielsweise Braunhirse, Hafer, Gerste, Schachtelhalmtee oder siliziumhaltige Nahrungsergänzungen.

\section{Säure-Basen-Verhältnis}

Die geläufigen Grundnahrungsmittel (Fleisch, Milchprodukte, Getreideprodukte/industriell verarbeitete tierische Produkte/Fertiggerichte) führen im Organismus gerade in Kombination mit Zucker zu einem Säureüberschuss, der durch die basischen Mineralstoffe wie Kalzium und Magnesium neutralisiert werden muss. Wenn diese nur in unzureichender Menge in der täglichen Nahrung vorhanden sind, werden die erforderlichen Mineralstoffe aus den Knochen entwendet und der Knochen kann entmineralisiert werden, so eine Theorie. Tierisches Protein in Milchprodukten kann zu einer erhöhten Kalziumausscheidung im Urin führen, da schwefelhaltige Aminosäuren und Phosphat zu vermehrter Kalziumausscheidung beitragen. Ob die Kalziumausscheidung den Knochenabbau überhaupt beeinflusst, ist zurzeit nicht

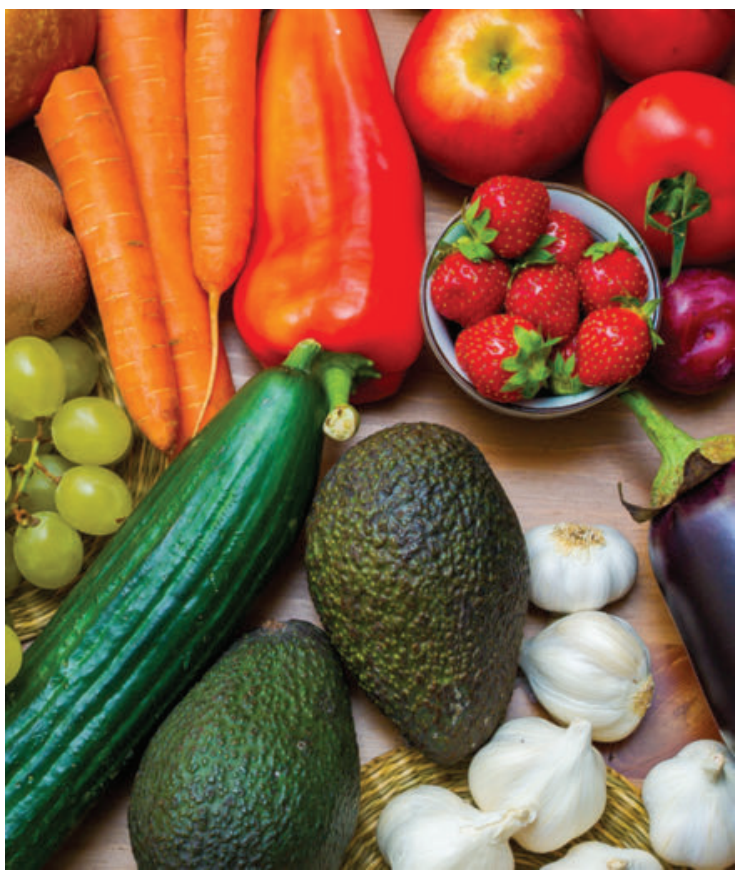

In der Heilungsphase ist Gemüse Milch- und Fleischprodukten vorzuziehen. (Quelle: napocska/ Shutterstock. com) 
ausreichend wissenschaftlich untersucht. Gemüse kann der Säurelast tierischer Lebensmittel entgegenwirken.

\section{Fazit}

Zusammenfassend ist eine gesunde, ausgewogene und vollwertige Ernährung wichtig für die Bruchheilung. Dabei kann eine milch- und fleischfreie Ernährung häufig besser dazu beitragen, da hier gerade die richtige Kombination der Nährstoffe vorhanden ist und dadurch die Aufnahme in den Knochen verbessert wird.

Verzichtet werden sollte unter anderem auf zu viel Phosphat, Alkohol, Koffein, Zucker, Salz, Eiweiß und Fett, da es unter anderem zu einer erhöhten Ausscheidung von Kalzium und Magnesium oder zu einer Resorptionsstörung im Darm führen kann.

\section{Autorinnen/Autoren}

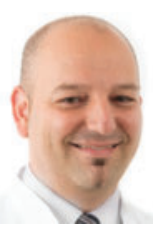

\section{Dr. Harun Seyhan}

Facharzt für Plastische und Ästhetische Chirurgie mit der Zusatzbezeichnung für Handchirurgie. Er ist leitender Oberarzt und ständiger Vertreter des Chefarztes im Krankenhaus KölnMerheim in der Klinik für Plastische Chirurgie, Handchirurgie und Schwerbrandverletztenzentrum - Kliniken der Stadt Köln gGmbH, Klinikum der Universität Witten/Herdecke und Lehrstuhl für Plastische Chirurgie der Universität Witten/Herdecke.

\section{Korrespondenzadresse}

\section{Harun Seyhan}

Krankenhaus Köln-Merheim

Abteilung für Plastische Chirurgie

Ostmerheimer Str. 200

51109 Köln

E-Mail: SeyhanH@kliniken-koeln.de

Literatur

[1] Osteoporose Selbsthilfegruppen Dachverband e. V. Calcium: Der wichtigste Baustein. Im Internet: www.osd-ev.org/osteo porose/knochen/calcium/; Stand: 12.11.2019 